\title{
DISCRETE COMPLEMENTED ALGEBRA
}

\section{PARFENY P. SAWOROTNOW}

I. Recently [5] the author introduced the concept of a discrete $H^{*}$-algebra. A two-sided $H^{*}$-algebra $A$ is called discrete if each invariant closed ideal in $A$ contains a two-sided projection (a two-sided projection is an idempotent which is both left and right self-adjoint). (An ideal $I$ is said to be invariant if $I^{r}=\left\{x^{r} \mid x \in I\right\}=\left\{x^{l} \mid x \in I\right\}$ $=I^{l}$.) It was shown that a simple proper discrete two-sided $H^{*}$. algebra is isomorphic to a canonical algebra. (A canonical algebra is an algebra of the type described in the example below with the additional assumption that the sequence $\{t(i)\}$ is bounded.)

The intent of this note is to obtain a similar result for complemented algebras. It is necessary to use a different technique since the involutions are not defined everywhere in the case of a general complemented algebra. In particular we define an invariant ideal in a different (but equivalent) fashion.

We continue to use terminology and notation of [3], [4], and [5]. In particular $l(R)(r(L))$ will denote the left (right) annihilator of a right (left) ideal $R(L)$ in an algebra; $R^{p}\left(L^{p}, l(R)^{p}, r(L)^{p}\right)$ will denote the orthogonal complement of $R(L, l(R), r(L))$.

II. Let $A$ be a Banach algebra with a Hilbert space norm. $A$ is called a complemented algebra [3] if the orthogonal complement of each right (left) ideal is again a right (left) ideal.

Definition 1. A closed right (left) ideal $R(L)$ in a complemented algebra $A$ is said to be invariant if $l\left(R^{p}\right)=l(R)^{p}\left(r\left(L^{p}\right)=r(L)^{p}\right)$ i.e. the orthogonal complement of the (appropriate) annihilator of an ideal coincides with the (appropriate) annihilator of its orthogonal complement.

From corollary to Lemma 3 in [5] it follows that in the case of a two-sided $H^{*}$-algebra this definition of an invariant ideal is equivalent to the definition given in [5]. Note also that an ideal $I$ is invariant if and only if its orthogonal complement $I^{p}$ is invariant.

It was established in [4] that in a semisimple complemented algebra $A$ there are two involutions, $x \rightarrow x^{r}$ and $x \rightarrow x^{l}$, defined on dense subsets of $A$, such that $(y x, z)=\left(y, z x^{r}\right)$ and $(x y, z)=\left(y, x^{l} z\right)$ for all $y, z \in A$. If $A$ is not semisimple we cannot speak about involutions since the right (left) adjoint $x^{r}\left(x^{l}\right)$ of a member $x$ of $A$ does not have to be unique. However, even in this case we may taik about a right

Received by the editors June 17, 1965. 
(left) selfadjoint element: $x \in A$ is right (left) selfadjoint if $(y x, z)$ $=(y, z x)((x y, z)=(y, x z)$ for all $y, z \in A$.) A right (left) selfadjoint (nonzero) idempotent is called a right (left) projection [3]. A twosided projection is a (nonzero) idempotent which is both right and left selfadjoint [5, definition after Lemma 3].

Definition 2. A complemented algebra $A$ is said to be discrete if each invariant closed right ideal in $A$ contains a two-sided projection.

EXAMPLE. Let $J$ be an arbitrary set and let $t(i)$ be a real-valued function defined on $J$ such that $1 \leqq t(i)$ for each $i \in J$. Let $M$ be the set of all complex-valued matrices $x=\left(x_{i j}\right)$ defined on $J \times J$ such that $\sum_{i, j} t(i)\left|x_{i j}\right|{ }^{2} t(j)$ converges. Then $M$ is a complemented algebra in the ordinary operations of matrices and the scalar product

$$
(x, y)=\sum_{i, j} t(i) x_{i j} \bar{y}_{i j} t(j)
$$

To see that $M$ is a complemented algebra, note that $M$ contains a dense subset of elements $x$ having a right adjoint $x^{r}\left(x_{i j}^{r}=t(i) / t(j) \bar{x}_{j i}\right)$ and a dense subset of elements $y$ having a left adjoint $y^{l}\left(y_{i j}^{l}\right.$ $\left.=t(j) / t(i) \bar{y}_{j i}\right)$. Let us show that the algebra $M$ is discrete. For each $i \in J$ let $e^{i}$ be a member of $M$ such that $e_{k j}^{i}=1$ if $k=j=i$ and $e_{k j}^{i}=0$ for every other pair $k, j$. Then $\left\{e^{i}\right\}_{i \in J}$ is a set of mutually orthogonal minimal two-sided projections such that $M=\sum_{i \in J} e^{i} M=\sum_{i \in J} M e^{i}$.

Let $R$ be a closed invariant right ideal in $M$. Let $e \in\left\{e^{i}\right\}_{i \in J}$ be such that $e R \neq 0$. Let us write $e=a_{1}+a_{2}=b_{1}+b_{2}$ with $a_{1} \in R, a_{2} \in R^{p}$, $b_{1} \in l\left(R^{p}\right)=l(R)^{p}, \quad b_{2} \in l(R)$. Then $a_{1} \neq 0$ (otherwise $e R=0$ ). From $e=e e=\left(a_{1}+a_{2}\right) e=a_{1} e+a_{2} e$ we conclude that $a_{1}=a_{1} e, a_{2}=a_{2} e$; similarly $b_{1}=e b_{1}, b_{2}=e b_{2}$. From Theorem 1 of [4] it follows that $b_{1}, b_{2}$ have lef $t$ adjoints; for the same reason $a_{1}, a_{2}$ have right adjoints. In fact, as it can be easily seen, $a_{1}^{r} \in l(R)^{p}=l\left(R^{p}\right), a_{2}^{r} \in l(R)$ (if $x \in l(R)$ then $\left(a_{1}^{r}, x\right)$ $=\left(e a_{1}^{r}, x\right)=\left(e, x a_{1}\right)=0$; if $x \in l\left(R^{p}\right)=l(R)^{p}$ then $\left.\left(a_{2}^{r}, x\right)=\left(e, x a_{2}\right)=0\right)$. But $a_{1}^{r}+a_{2}^{r}=e^{r}=e=b_{1}+b_{2}$ and so $a_{1}^{r}=b_{1}, a_{2}^{r}=b_{2}$. By symmetry we have $b_{1}^{l}=a_{1}, b_{2}^{l}=a_{2}$. Thus $b_{1}=a_{1}^{r}=a_{1}^{l}$ and so $a_{1} b_{1}=a_{1} a_{1}^{r}=a_{1} a_{1}^{l}$ is an element in $R$ which is both right and left selfadjoint. Following the proof of Lemma 1 on p. 101 of [2] we now show that $R$ contains a two-sided projection.

III. Lemma 1. Let $\left\{e_{\alpha}\right\}$ be a sequence of mutually orthogonal twosided projections in a proper complemented algebra $A$. ( $A$ complemented algebra $A$ is proper if $r(A)=(0)\left[3\right.$, p. 50].) Then the ideals $R=\sum_{\alpha} e_{\alpha} A$ and $L=\sum_{\alpha} A e_{\alpha}$ are invariant.

Proof. First note that $l(R)=\bigcap_{\alpha} l\left(e_{\alpha}\right)$ and $r(L)=\bigcap_{\alpha} r\left(e_{\alpha}\right)$. By direct verification we see that 
(1) $R^{p}=r(L)$ and that

(2) $l(R)=L^{p}$

(for example $x \in R^{p}$ would imply $\left\|e_{\alpha} x\right\|^{2}=\left(x, e_{\alpha} x\right)=0$ for each $\alpha$ and $x \in r(L)$ would imply $\left(x, e_{\alpha} y\right)=\left(e_{\alpha} x, y\right)=0$ for each $\alpha$ and each $\left.y \in A\right)$. From (1) we conclude that $L \subset l\left(R^{p}\right)$; from (2) one can see that $x \in L^{p} \cap l\left(R^{p}\right)$ would imply $x R=0$ and $x R^{p}=0$ which is only possible if $x=0$ (note that $l(A)=0$ also [3, p. 50]). Thus $l\left(R^{p}\right)=l(R)^{p}(=L)$. Similarly $r\left(L^{p}\right)=r(L)^{p}$.

In the next lemma and the theorem after it, we will use the term "simple" to mean "simple and semisimple" $[4$, p. 56]. (Note that in our terminology each simple algebra is proper $[3$, p. 50].)

Lemma 2. Each invariant right ideal $R$ in a simple discrete complemented algebra $A$ contains a minimal two-sided projection. The same is true about left ideals.

Proof. Let $e$ be a two-sided projection in $R$. If we write $e$ as a sum of mutually orthogonal primitive (minimal) left projections and apply Theorem 1 of [4] we can conclude that each element in $e A$ has a left adjoint in $A e$. Similarly each element in $A e$ has a right adjoint in $e A$. Thus $A_{0}=e A e$ is a proper two-sided $H^{*}$-algebra. It is finite dimensional ( $e$ is the identity for $A_{0}$ ) and so by Theorem 3 of [5] $A_{0}$ contains a minimal two-sided projection. Lemma 2 now follows from the fact that $A_{0} \subset R$.

THEOREM. Each simple discrete complemented algebra $A$ is isomorphic to an algebra $M$ of the type described in the example above.

Proof. From the above lemmas it follows that $A$ contains a (maximal) set $\left\{e_{i}\right\}_{i \in J}$ of mutually orthogonal minimal projections such that $A=\sum_{i} e_{i} A=\sum_{i} A e_{i}=\sum_{i, j} e_{i} A e_{j}$. Using this set we can proceed as in the proof of Theorem 4 in [5] (in the present case we do not have to prove that the function $t(i)$ is bounded above) (note also that each element in $e_{i} A e_{j}$ has both right and left adjoints).

\section{REFERENCES}

1. W Ambrose, Structure theorems for a special class of Banach algebras, Trans. Amer. Math. Soc. 57 (1945), 364-386.

2. L. H. Loomis, An introduction to abstract harmonic analysis, Van Nostrand, New York, 1953.

3. P. P. Saworotnow, On a generalization of the notion of $H^{*}$-algebra, Proc. Amer. Math. Soc. 8 (1957), 49-55.

4. - On the imbedding of a right complemented algebra into Ambrose's $H^{*}$. algebra, Proc. Amer. Math. Soc. 8 (1957), 56-62.

5. —, On two-sided $H^{*}$-algebras, Pacific J. Math. 16 (1966), No. 1.

The Catholic University of America 